EPJ manuscript No.

(will be inserted by the editor)

\title{
On the chiral effective meson-baryon Lagrangian at third order *
}

\author{
Matthias Frink $^{1}$ a and Ulf-G. Meißner ${ }^{12}$ b \\ 1 Helmholtz-Institut für Strahlen- und Kernphysik (Theorie), Universität Bonn, Nußallee 14-16, D-53115 Bonn, Germany \\ 2 Forschungszentrum Jülich, Institut für Kernphysik (Theorie), D-52425 Jülich, Germany
}

Received: date / Revised version: date

Abstract. We show that the recently constructed complete and "minimal" third order meson-baryon effective chiral Lagrangian can be further reduced from 84 to 78 independent operators.

PACS. 12.39.Fe Chiral Lagrangians

Recently, Oller et al. [1] have presented a complete and "minimal" chiral effective Lagrangian for three flavor baryon chiral perturbation theory to third order in the chiral expansion, utilizing the methods outlined in Ref. [2] for constructing the fourth order two flavor effective Lagrangian. The first attempt to construct the $\mathrm{SU}(3)$ meson-baryon Lagrangian goes back to Krause [3]. However, he made no attempt at minimizing the number of independent operators at third order. We are presently constructing the minimal fourth order SU(3) Lagrangian for Goldstone bosons coupled to the ground state baryon octet and external sources. As a by-product we show in this note that the number of 84 independent third order operators in Ref. [1] can further be reduced by a combination of using certain Cayley-Hamilton relations and the baryon equations of motion.

The chiral effective Lagrangian can be constructed by writing down the pertinent building blocks to generate chirally invariant operators at a given order in the chiral expansion. The building blocks are expressed in terms of the standard meson matrices $U, u^{2}=U$, the baryon matrix $B$, and the external sources $\chi=s+i p, r_{\mu}, l_{\mu}$ :

$$
\begin{aligned}
u_{\mu} & =i u^{\dagger} \nabla_{\mu} U u^{\dagger}=-i u \nabla_{\mu} U^{\dagger} u \\
\chi^{ \pm} & =u \chi^{\dagger} u \pm u^{\dagger} \chi u^{\dagger} \\
F_{\mu \nu}^{ \pm} & =u^{\dagger} R_{\mu \nu} u \pm u L_{\mu \nu} u^{\dagger} .
\end{aligned}
$$

Here

$$
\begin{aligned}
\nabla_{\mu} U & =\partial_{\mu} U-i r_{\mu} U+i U l_{\mu}, \\
R_{\mu \nu} & =\partial_{\mu} r_{\nu}-\partial_{\nu} r_{\mu}-i\left[r_{\mu}, r_{\nu}\right],
\end{aligned}
$$

* This research is part of the EU Integrated Infrastructure Initiative Hadron Physics Project under contract number RII3CT-2004-506078. Work supported in part by DFG (SFB/TR 16 "Subnuclear Structure of Matter").

a Electronic address: mfrink@itkp.uni-bonn.de

b Electronic address: meissner@itkp.uni-bonn.de in terms of the commutator [, ], and similarly for $l_{\mu}$. The covariant derivative acting on any field $X=B, u_{\mu}, \ldots$ reads

$$
\left[D_{\mu}, X\right]=\partial_{\mu} X+\left[\Gamma_{\mu}, X\right],
$$

where $\Gamma_{\mu}$ is given by

$$
\Gamma_{\mu}=\frac{1}{2}\left[u^{\dagger}, \partial_{\mu} u\right]-\frac{i}{2}\left(u^{\dagger} r_{\mu} u+u l_{\mu} u^{\dagger}\right) .
$$

To proceed, one has to assign a chiral dimension to these building blocks. We work here in standard chiral perturbation theory, that is all fields are of order $q^{0}$ (where $q$ is the small expansion parameter), and so are derivatives acting on baryon fields. Derivatives applied to meson or external fields, vector and axial-vector sources are $\mathcal{O}(q)$, and the field strength tensors as well as the scalar and pseudoscalar sources are $\mathcal{O}\left(q^{2}\right)$. The latter assignment reflects the fact that the Goldstone boson masses squared are proportional to the quark masses at leading order in the chiral expansion. Consequently, the leading order effective Lagrangian is of chiral order one, next-to-leading order corrections are of order two, and the first loop contributions appear at third order together with the local contact interactions that are considered in this note. Thus, the effective Lagrangian takes the form

$$
\mathcal{L}_{M B}=\mathcal{L}_{M B}^{(1)}+\mathcal{L}_{M B}^{(2)}+\mathcal{L}_{M B}^{(3)}+\ldots,
$$

where the ellipsis denotes higher order terms.

We skip here any technical details how to construct the effective Lagrangian from the building blocks, apart from certain relations that are utilized to further minimize the number of third order operators. For these details, we refer to Refs. $[1,2,4]$. We fully agree with $\mathcal{L}_{M B}^{(2)}$ in Eq. (5.1) of Ref. [1]. In $\mathcal{L}_{M B}^{(3)}$ in Eq. (5.2), however, six of the 84 terms listed there are redundant by one of the following mechanisms:

- SU(3) Cayley-Hamilton trace identities; 
- the lowest-order baryon equation of motion $i \gamma^{\mu}\left[D_{\mu}, B\right]=m_{0} B+\mathcal{O}(q)$, with $m_{0}$ the octet baryon mass in the chiral limit.

The Cayley-Hamilton identities allow to discard five of the structures in $\mathcal{L}_{M B}^{(3)}$ in Ref. [1], e.g. $\mathrm{O}_{20}, \mathrm{O}_{21}, \mathrm{O}_{22}, \mathrm{O}_{35}, \mathrm{O}_{36}$. The details of this procedure are spelled out in App. A

We now give details how a further reduction of the number of terms is achieved. By means of the lowestorder baryon equation of motion monomials containing $\gamma^{\mu}\left[D_{\mu}, B\right]$ can be reduced to the corresponding structures without the covariant derivative and higher-order contributions. Revealing prospective Lagrangian terms to be redundant means exposing those $\gamma^{\mu}\left[D_{\mu}, B\right]$ structures where-ever they occur hidden in the Clifford-algebra. In Ref. [1] this procedure is implemented in terms of the relations Eqs. (4.4)-(4.10). Some of these equations are, however, interrelated and their full content cannot be unraveled by treating them as separate identities. To illustrate this feature consider the following third-order combination of building blocks comprising two mesonic fields $u_{\lambda}, u_{\rho}$ together with two covariant derivatives, where one derivative $\tilde{D}_{\nu}$ acts on meson fields, as indicated by the tilde symbol, and the other one, $D_{\mu}$, acts on the baryon matrix:

$$
\left(u_{\lambda}, u_{\rho}, \tilde{D}_{\nu}, D_{\mu}\right) \leftrightarrow\left(\varepsilon^{\mu \nu \rho \lambda}, g^{\mu \nu} \sigma^{\rho \lambda}, g^{\mu \nu} g^{\rho \lambda}, \ldots\right)
$$

The left-hand-side gives rise to invariants by combing meson and baryon matrices into products of traces and applying the covariant derivatives. Having one derivative each act on the two types of fields involved, one specific trace configuration generates contributions referred to as $D_{\mu} \tilde{D}_{\nu}$-terms and accordingly for $\tilde{D}_{\mu} D_{\nu}$. As suggested by the right-hand-side of Eq. (6), the four Lorentz indices can be contracted with the totally anti-symmetric tensor $\varepsilon$, a product of one metric tensor $g$ and one antisymmetric Clifford-algebra element $\sigma$, or else two metric tensors, where the ellipsis indicates that any ordering of the indices is possible, up to occurring index symmetries. Consider now the two structures $\sigma^{\mu \rho} g^{\nu \lambda}, g^{\mu \rho} \sigma^{\nu \lambda}$. The following identities among Clifford-algebra elements,

$$
\begin{aligned}
g^{\mu \rho} g^{\nu \lambda} & =-i \sigma^{\mu \rho} g^{\nu \lambda}+\gamma^{\rho} \gamma^{\mu} g^{\nu \lambda} \\
& =-i g^{\mu \rho} \sigma^{\nu \lambda}+g^{\mu \rho} \gamma^{\lambda} \gamma^{\nu}
\end{aligned}
$$

are the basis of Eq. (4.4) in Ref. [1], which implies that $\sigma^{\mu \rho} g^{\nu \lambda}$ in connection with the $D_{\mu} \tilde{D}_{\nu}$-terms is eliminated in favor of $g^{\mu \rho} g^{\nu \lambda}$, and accordingly for $g^{\mu \rho} \sigma^{\nu \lambda}$ contracted with the $\tilde{D}_{\mu} D_{\nu^{-}}$-configurations. If all $D_{\mu} \tilde{D}_{\nu^{-}}$as well as $\tilde{D}_{\mu} D_{\nu}$-terms are summed up, the covariant derivatives can be reshuffled by a total derivative argument to act on baryon fields only, thus producing a $D_{\mu} D_{\nu}$-structure. $\sigma^{\mu \rho} g^{\nu \lambda}$ contracted with this structure is related by the eliminating index $\mu$ to $g^{\mu \rho} g^{\nu \lambda}$, which, in turn, can be connected to the corresponding contribution including $g^{\mu \rho} \sigma^{\nu \lambda}$ by means of manipulations in the index $\nu$. Therefore, the above $D_{\mu} D_{\nu}$-term contracted with the difference $\sigma^{\mu \rho} g^{\nu \lambda}-$ $g^{\mu \rho} \sigma^{\nu \lambda}$ can be ignored making use of the baryon equations of motion. As $D_{\mu} D_{\nu}$ is symmetric in the indices $\mu$ and $\nu$, this leads to the elimination of one $D_{\mu} \tilde{D}_{\nu^{-}}$or
$\tilde{D}_{\mu} D_{\nu}$-contribution associated with a field configuration anti-symmetric in the indices $\rho$ and $\lambda$ contracted with, say, $\sigma^{\mu \rho} g^{\nu \lambda}$. Since this Clifford structure reduces the combination $\left[D_{\mu}, u_{\rho}\right]$ via the relation $\left[D_{\mu}, u_{\rho}\right]-\left[D_{\rho}, u_{\mu}\right]=F_{\mu \rho}^{-}$, we are left with two prospective terms including $\left[D_{\mu}, u_{\lambda}\right]$ which are not independent: one of the monomials $O_{9}, O_{10}$ in $\mathcal{L}_{M B}^{(3)}$ from Ref. [1] is redundant. Thus, together with the five operators removed by use of the Cayley-Hamilton relations, we have reduced the number of independent operators from 84 to 78 .

We furthermore remark that $O_{41}$ in Ref. [1] has the wrong behavior under charge conjugation. Adopting the notation used there the operation of charge conjugation is given by:

$$
\begin{aligned}
& \left\langle\bar{B} \sigma^{\lambda \tau} D_{\rho} B\right\rangle\left\langle u^{\mu} u^{\nu} u^{\rho}\right\rangle \varepsilon_{\mu \nu \lambda \tau} \\
& \quad \stackrel{\mathcal{C}}{\longrightarrow}-\left\langle\bar{B} \overleftarrow{D}_{\rho} \sigma^{\lambda \tau} B\right\rangle\left\langle u^{\nu} u^{\mu} u^{\rho}\right\rangle \varepsilon_{\mu \nu \lambda \tau}
\end{aligned}
$$

where use has been made of the cyclic property of the trace. Accounting for the inverted order of fields in the final trace by an index exchange induces a change in the relative sign. The proper charge conjugation invariant structure is of higher order and the remark stating an abnormal non-relativistic power counting behavior for this term is then rendered pointless.

Similarly, the ordering of indices in the monomials $O_{31}$, $\mathrm{O}_{33}$, and $\mathrm{O}_{34}$ does not match the conditions imposed by charge conjugation. In the notation used there, the invariant assignment of indices e.g. for $O_{31}$ reads, given the symmetric product of covariant derivatives:

$$
\left\langle\bar{B} \gamma_{5} \gamma_{\mu} D_{\nu \rho} B u^{\mu} u^{\nu} u^{\rho}\right\rangle+\left\langle\bar{B} \overleftarrow{D}_{\nu \rho} \gamma_{5} \gamma_{\mu} B u^{\nu} u^{\rho} u^{\mu}\right\rangle
$$

We finally present a list of the Lagrangian invariants where the above corrections have been accounted for:

$$
\mathcal{L}_{M B}^{(3)}=\sum_{i=1}^{78} d_{i} O_{i}^{(3)} .
$$

The explicit terms are collected in Tab. 11 The basis chosen is different from the one in Ref. [1]. All third-order LECs $d_{i}$ accompanying the operators $O_{i}^{(3)}$ are of dimension mass $^{-2}$. Using the same techniques, we are presently constructing the complete and minimal fourth order Lagrangian for $\mathrm{SU}(3)$ baryon chiral perturbation theory. The terms are needed for any complete one-loop calculation (for the example of the baryon masses at fourth order, see e.g. Ref. [5] and references therein).

\section{Acknowledgements}

We are grateful to Bastian Kubis for useful comments.

\section{A Cayley-Hamilton relations}

The following notation is introduced to classify CayleyHamilton identities: a relation consisting of a vanishing 
sum of traces and products thereof is referred to by the term in the sum that contains the trace extending over the maximum number of matrices. Applied to the case of three matrix-valued fields $A_{1}, A_{2}, A_{3}$ included, together with the baryon fields $\bar{B}, B$, in one or several traces, 15 independent identities result for complex $3 \times 3$ matrices. Formulated as invariants with definite behavior under charge conjugation, they fall into $\mathrm{C}$-even and C-odd relations, respectively. These are listed below together with a possible choice of structures thus rendered redundant. Here, $A_{1} A_{2} A_{3}+$ perm. stands for the product of the three matrices in all possible orders given by the six possible permutations of the indices $\{1,2,3\}$ summed up. [ , ] and $\{$,$\} stand for commutators and anti-commutators, re-$ spectively. $\Gamma$ represents an element of the Clifford-algebra.

C-even:

$$
\operatorname{Tr}\left\{\left(\bar{B} B A_{1}+\text { perm. }\right) A_{2}\right\} \operatorname{Tr}\left\{A_{3}\right\}
$$

is used to eliminate

$$
\begin{aligned}
& \operatorname{Tr}\left\{\bar{B} A_{1}\right\} \Gamma \operatorname{Tr}\left\{A_{2} B\right\} \operatorname{Tr}\left\{A_{3}\right\} \\
& \quad+\operatorname{Tr}\left\{\bar{B} A_{2}\right\} \Gamma \operatorname{Tr}\left\{A_{1} B\right\} \operatorname{Tr}\left\{A_{3}\right\},
\end{aligned}
$$

and accordingly for $\operatorname{Tr}\left\{\left(\bar{B} B A_{1}+\right.\right.$ perm. $\left.) A_{3}\right\} \operatorname{Tr}\left\{A_{2}\right\}$ and $\operatorname{Tr}\left\{\left(\bar{B} B A_{2}+\right.\right.$ perm. $\left.) A_{3}\right\} \operatorname{Tr}\left\{A_{1}\right\}$.

$$
\begin{aligned}
& \operatorname{Tr}\left\{\left(\bar{B} B A_{1}+\text { perm. }\right)\left\{A_{2}, A_{3}\right\}\right\}, \\
& \operatorname{Tr}\left\{\left(\bar{B} B A_{2}+\text { perm. }\right)\left\{A_{1}, A_{3}\right\}\right\}, \\
& \operatorname{Tr}\left\{\left(\bar{B} B A_{3}+\text { perm. }\right)\left\{A_{1}, A_{2}\right\}\right\}, \\
& \operatorname{Tr}\left\{\left(A_{1} A_{2} A_{3}+\text { perm. }\right)[\bar{B}, B]\right\}, \\
& \operatorname{Tr}\left\{\left(B A_{2} A_{3}+\text { perm. }\right)\left[\bar{B}, A_{1}\right]\right\} \\
& \quad+\operatorname{Tr}\left\{\left(\bar{B} A_{2} A_{3}+\text { perm. }\right)\left[A_{1}, B\right]\right\}, \\
& \operatorname{Tr}\left\{\left(B A_{1} A_{3}+\text { perm. }\right)\left[\bar{B}, A_{2}\right]\right\} \\
& \quad+\operatorname{Tr}\left\{\left(\bar{B} A_{1} A_{3}+\text { perm. }\right)\left[A_{2}, B\right]\right\}, \\
& \operatorname{Tr}\left\{\left(B A_{1} A_{2}+\text { perm. }\right)\left\{\bar{B}, A_{3}\right\}\right\} \\
& \quad+\operatorname{Tr}\left\{\left(\bar{B} A_{1} A_{2}+\text { perm. }\right)\left\{A_{3}, B\right\}\right\}
\end{aligned}
$$

is used to eliminate

$$
\begin{aligned}
& \operatorname{Tr}\left\{\bar{B} \Gamma\left\{A_{1}, B\right\}\right\} \operatorname{Tr}\left\{A_{2} A_{3}\right\}, \\
& \operatorname{Tr}\left\{\bar{B} \Gamma\left\{A_{2}, B\right\}\right\} \operatorname{Tr}\left\{A_{1} A_{3}\right\}, \\
& \operatorname{Tr}\left\{\bar{B} \Gamma\left\{A_{3}, B\right\}\right\} \operatorname{Tr}\left\{A_{1} A_{2}\right\}, \\
& \operatorname{Tr}\left\{\bar{B} \Gamma\left[A_{1}, B\right]\right\} \operatorname{Tr}\left\{A_{2} A_{3}\right\}, \\
& \operatorname{Tr}\left\{\bar{B} \Gamma\left[A_{2}, B\right]\right\} \operatorname{Tr}\left\{A_{1} A_{3}\right\}, \\
& \operatorname{Tr}\left\{\bar{B} \Gamma\left[A_{3}, B\right]\right\} \operatorname{Tr}\left\{A_{1} A_{2}\right\}, \\
& \operatorname{Tr}\left\{\bar{B} A_{3}\right\} \Gamma \operatorname{Tr}\left\{\left\{A_{1}, A_{2}\right\} B\right\} \\
& \quad+\operatorname{Tr}\left\{\bar{B}\left\{A_{1}, A_{2}\right\}\right\} \Gamma \operatorname{Tr}\left\{A_{3} B\right\},
\end{aligned}
$$

where the final relation is understood in its totally symmetric combination.
C-odd:

$$
\begin{aligned}
\operatorname{Tr} & \left\{\left(\bar{B} B A_{1}+\text { perm. }\right)\left[A_{2}, A_{3}\right]\right\}, \\
\operatorname{Tr} & \left\{\left(\bar{B} B A_{2}+\text { perm. }\right)\left[A_{1}, A_{3}\right]\right\}, \\
\operatorname{Tr} & \left\{\left(\bar{B} B A_{3}+\text { perm. }\right)\left[A_{1}, A_{2}\right]\right\}, \\
\operatorname{Tr} & \left\{\left(B A_{2} A_{3}+\text { perm. }\right)\left\{\bar{B}, A_{1}\right\}\right\} \\
& -\operatorname{Tr}\left\{\left(\bar{B} A_{2} A_{3}+\text { perm. }\right)\left\{A_{1}, B\right\}\right\}, \\
\operatorname{Tr} & \left\{\left(B A_{1} A_{3}+\text { perm. }\right)\left\{\bar{B}, A_{2}\right\}\right\} \\
& -\operatorname{Tr}\left\{\left(\bar{B} A_{1} A_{3}+\text { perm. }\right)\left\{A_{2}, B\right\}\right\}
\end{aligned}
$$

is used to eliminate

$$
\begin{aligned}
& \operatorname{Tr}\left\{\bar{B} A_{1}\right\} \Gamma \operatorname{Tr}\left\{\left[A_{2}, A_{3}\right] B\right\} \\
& \quad+\operatorname{Tr}\left\{\bar{B}\left[A_{2}, A_{3}\right]\right\} \Gamma \operatorname{Tr}\left\{A_{1} B\right\}, \\
& \operatorname{Tr}\left\{\bar{B} A_{2}\right\} \Gamma \operatorname{Tr}\left\{\left[A_{1}, A_{3}\right] B\right\} \\
& \quad+\operatorname{Tr}\left\{\bar{B}\left[A_{1}, A_{3}\right]\right\} \Gamma \operatorname{Tr}\left\{A_{2} B\right\}, \\
& \operatorname{Tr}\left\{\bar{B} A_{3}\right\} \Gamma \operatorname{Tr}\left\{\left[A_{1}, A_{2}\right] B\right\} \\
& \quad+\operatorname{Tr}\left\{\bar{B}\left[A_{1}, A_{2}\right]\right\} \Gamma \operatorname{Tr}\left\{A_{3} B\right\}, \\
& \operatorname{Tr}\left\{\bar{B} A_{1}\right\} \Gamma \operatorname{Tr}\left\{\left\{A_{2}, A_{3}\right\} B\right\} \\
& \quad-\operatorname{Tr}\left\{\bar{B}\left\{A_{2}, A_{3}\right\}\right\} \Gamma \operatorname{Tr}\left\{A_{1} B\right\}, \\
& \operatorname{Tr}\left\{\bar{B} A_{2}\right\} \Gamma \operatorname{Tr}\left\{\left\{A_{1}, A_{3}\right\} B\right\} \\
& \quad-\operatorname{Tr}\left\{\bar{B}\left\{A_{1}, A_{3}\right\}\right\} \Gamma \operatorname{Tr}\left\{A_{2} B\right\},
\end{aligned}
$$

where the final two relations are understood in their $1 \leftrightarrow 3$ anti-symmetric and $2 \leftrightarrow 3$ anti-symmetric combinations, respectively.

\section{References}

1. J. A. Oller, M. Verbeni and J. Prades, arXiv:hep-ph/0608204

2. N. Fettes, U.-G. Meißner, M. Mojžiš and S. Steininger, Annals Phys. 283 (2000) 273 [Erratum-ibid. 288 (2001) 249] arXiv:hep-ph/0001308.

3. A. Krause, Helv. Phys. Acta 63 (1990) 3.

4. M. Frink and U.-G. Meißner, "The chiral effective mesonbaryon Lagrangian at fourth order," in preparation.

5. M. Frink and U.-G. Meißner, JHEP 0407 (2004) 028 arXiv:hep-lat/0404018. 
Matthias Frink, Ulf-G. Meißner: On the chiral effective meson-baryon Lagrangian at third order

\begin{tabular}{|c|c|}
\hline$i$ & $O_{i}^{(3)}$ \\
\hline 1 & $m \operatorname{Tr}\left\{\bar{B} \gamma^{5}\left[\gamma^{-} B\right]\right\}$ \\
\hline 2 & $\begin{array}{l}m_{0} \operatorname{Ir}\left\{B \gamma^{\gamma}\{\chi, B]\right\} \\
m_{0} \operatorname{Tr}\left\{\bar{B} \gamma^{5}\left\{\chi^{-}, B\right\}\right\}\end{array}$ \\
\hline 3 & $m_{0} \operatorname{Tr}\left\{\bar{B} \gamma^{5} B\right\} \operatorname{Tr}\left\{\chi^{-}\right\}$ \\
\hline 4 & $i\left(\operatorname{Tr}\left\{\bar{B} \varepsilon^{\mu \nu \rho \tau} \gamma_{\tau}\left[\left[u_{\mu}, u_{\nu}\right],\left[u_{\rho}, B\right]\right]\right\}+\operatorname{Tr}\left\{\bar{B}^{\mu \nu \rho \tau} \gamma_{\tau}\left[u_{\rho},\left[\left[u_{\mu}, u_{\nu}\right], B\right]\right]\right\}\right)$ \\
\hline 5 & $i\left(\operatorname{Tr}\left\{\bar{B} \varepsilon^{\mu \nu \rho \tau} \gamma_{\tau}\left[\left[u_{\mu}, u_{\nu}\right],\left\{u_{\rho}, B\right\}\right]\right\}+\operatorname{Tr}\left\{\bar{B} \varepsilon^{\mu \nu \rho \tau} \gamma_{\tau}\left\{u_{\rho},\left[\left[u_{\mu}, u_{\nu}\right], B\right]\right\}\right\}\right)$ \\
\hline 6 & $i\left(\operatorname{Tr}\left\{\bar{B} \varepsilon^{\mu \nu \rho \tau} \gamma_{\tau}\left\{\left[u_{\mu}, u_{\nu}\right],\left[u_{\rho}, B\right]\right\}\right\}+\operatorname{Tr}\left\{\bar{B} \varepsilon^{\mu \nu \rho \tau} \gamma_{\tau}\left[u_{\rho},\left\{\left[u_{\mu}, u_{\nu}\right], B\right\}\right]\right\}\right.$ \\
\hline 7 & $i\left(\operatorname{Tr}\left\{\bar{B} \varepsilon^{\mu \nu \rho \tau} \gamma_{\tau}\left\{\left[u_{\mu}, u_{\nu}\right],\left\{u_{\rho}, B\right\}\right\}\right\}+\operatorname{Tr}\left\{\bar{B} \varepsilon^{\mu \nu \rho \tau} \gamma_{\tau}\left\{u_{\rho},\left\{\left[u_{\mu}, u_{\nu}\right], B\right\}\right\}\right\}\right)$ \\
\hline 8 & $i \operatorname{Tr}\left\{\bar{B} \varepsilon^{\mu \nu \rho \tau} \gamma_{\tau} B\right\} \operatorname{Tr}\left\{u_{\mu}\left[u_{\nu}, u_{\rho}\right]\right\}$ \\
\hline 9 & $\operatorname{Tr}\left\{\bar{B} \gamma^{\mu} \gamma^{5}\left[u_{\nu},\left[u^{\nu},\left[u_{\mu}, B\right]\right]\right]\right\}+\operatorname{Tr}\left\{\bar{B} \gamma^{\mu} \gamma^{5}\left[u_{\mu},\left[u_{\nu},\left[u^{\nu}, B\right]\right]\right]\right\}$ \\
\hline 10 & $\operatorname{Tr}\left\{\bar{B} \gamma^{\mu} \gamma^{5}\left[u_{\nu},\left[u^{\nu},\left\{u_{\mu}, B\right\}\right]\right]\right\}+\operatorname{Tr}\left\{\bar{B} \gamma^{\mu} \gamma^{5}\left\{u_{\mu},\left[u_{\nu},\left[u^{\nu}, B\right]\right]\right\}\right\}$ \\
\hline 11 & $\operatorname{Tr}\left\{\bar{B} \gamma^{\mu} \gamma^{5}\left[u_{\nu},\left\{u^{\nu},\left[u_{\mu}, B\right]\right\}\right]\right\}+\operatorname{Tr}\left\{\bar{B} \gamma^{\mu} \gamma^{5}\left[u_{\mu},\left\{u_{\nu},\left[u^{\nu}, B\right]\right\}\right]\right\}$ \\
\hline 12 & $\operatorname{Tr}\left\{\bar{B} \gamma^{\mu} \gamma^{5}\left[u_{\nu},\left\{u^{\nu},\left\{u_{\mu}, B\right\}\right\}\right]\right\}+\operatorname{Tr}\left\{\bar{B} \gamma^{\mu} \gamma^{5}\left\{u_{\mu},\left\{u_{\nu},\left[u^{\nu}, B\right]\right\}\right\}\right\}$ \\
\hline 13 & $\operatorname{Tr}\left\{\underline{\bar{B}} \gamma^{\mu} \gamma^{5}\left\{u_{\nu},\left\{u^{\nu},\left[u_{\mu}, B\right]\right\}\right\}\right\}+\operatorname{Tr}\left\{\bar{B} \gamma^{\mu} \gamma^{5}\left[u_{\mu},\left\{u_{\nu},\left\{u^{\nu}, B\right\}\right\}\right]\right\}$ \\
\hline 14 & $\operatorname{Tr}\left\{\bar{B} \gamma^{\mu} \gamma^{5}\left\{u_{\nu},\left\{u^{\nu},\left\{u_{\mu}, B\right\}\right\}\right\}\right\}+\operatorname{Tr}\left\{\bar{B} \gamma^{\mu} \gamma^{5}\left\{u_{\mu},\left\{u_{\nu},\left\{u^{\nu}, B\right\}\right\}\right\}\right\}$ \\
\hline 15 & $\operatorname{Tr}\left\{\bar{B} \gamma^{\mu} \gamma^{5}\left[\left[u_{\nu},\left[u^{\nu}, u_{\mu}\right]\right], B\right]\right\}$ \\
\hline 16 & $\operatorname{Tr}\left\{\bar{B} \gamma^{\mu} \gamma^{5}\left\{\left[u_{\nu},\left[u^{\nu}, u_{\mu}\right]\right], B\right\}\right\}$ \\
\hline $\begin{array}{l}17 \\
18\end{array}$ & $\operatorname{Tr}\left\{\bar{B} u_{\nu}\right\} \gamma^{\mu} \gamma^{5} \operatorname{Tr}\left\{\left\{u^{\nu}, u_{\mu}\right\} B\right\}+\operatorname{Tr}\left\{\bar{B}\left\{u_{\nu}, u_{\mu}\right\}\right\} \gamma^{\mu} \gamma^{5} \operatorname{Tr}\left\{u^{\nu} B\right\}$ \\
\hline 19 & $\begin{array}{l}\operatorname{Tr}\left\{B u_{\nu}\right\} \gamma^{\mu} \gamma^{\nu} \operatorname{Tr}\left\{\left[u^{\nu}, u_{\mu}\right] B\right\}-\operatorname{Tr}\left\{B\left[u_{\nu}, u_{\mu}\right]\right\} \gamma^{\mu} \gamma^{\nu} \operatorname{Tr}\left\{u^{\nu} B\right\} \\
\operatorname{Tr}\left\{\bar{B} \gamma^{\mu} \gamma^{5} B\right\} \operatorname{Tr}\left\{u_{\mu} u_{\nu} u^{\nu}\right\}\end{array}$ \\
\hline 20 & $i \operatorname{Tr}\left\{\bar{B} \gamma^{\mu}\left[\left[\left[D_{\mu}, u_{\nu}\right], u^{\nu}\right], B\right]\right\}$ \\
\hline 21 & $i \operatorname{Tr}\left\{\bar{B} \gamma^{\mu}\left\{\left[\left[D_{\mu}, u_{\nu}\right], u^{\nu}\right], B\right\}\right\}$ \\
\hline 22 & $i\left(\operatorname{Tr}\left\{\bar{B} u_{\nu}\right\} \gamma^{\mu} \operatorname{Tr}\left\{\left[D_{\mu}, u^{\nu}\right] B\right\}-\operatorname{Tr}\left\{\bar{B}\left[D_{\mu}, u_{\nu}\right]\right\} \gamma^{\mu} \operatorname{Tr}\left\{u^{\nu} B\right\}\right)$ \\
\hline 23 & $\frac{i}{2 m_{0}}\left(\operatorname{Tr}\left\{\bar{B} \sigma^{\mu \nu}\left[\left[D_{\mu}, u_{\rho}\right],\left[u_{\nu},\left[D^{\rho}, B\right]\right]\right]\right\}+\operatorname{Tr}\left\{\bar{B} \sigma^{\mu \nu}\left[D_{\rho},\left[u_{\nu},\left[\left[D_{\mu}, u^{\rho}\right], B\right]\right]\right]\right\}\right)$ \\
\hline 24 & $\frac{i}{2 m_{0}}\left(\operatorname{Tr}\left\{\bar{B} \sigma^{\mu \nu}\left[\left[D_{\mu}, u_{\rho}\right],\left\{u_{\nu},\left[D^{\rho}, B\right]\right\}\right]\right\}+\operatorname{Tr}\left\{\bar{B} \sigma^{\mu \nu}\left[D_{\rho},\left\{u_{\nu},\left[\left[D_{\mu}, u^{\rho}\right], B\right]\right\}\right]\right\}\right.$ \\
\hline 25 & $\frac{i}{2 m_{0}}\left(\operatorname{Tr}\left\{\bar{B} \sigma^{\mu \nu}\left\{\left[D_{\mu}, u_{\rho}\right],\left\{u_{\nu},\left[D^{\rho}, B\right]\right\}\right\}\right\}+\operatorname{Tr}\left\{\bar{B} \sigma^{\mu \nu}\left[D_{\rho},\left\{u_{\nu},\left\{\left[D_{\mu}, u^{\rho}\right], B\right\}\right\}\right]\right\}\right)$ \\
\hline 26 & $\frac{i}{m_{0}}\left(\operatorname{Tr}\left\{\bar{B} \sigma^{\mu \nu}\left[D_{\rho}, B\right]\right\} \operatorname{Tr}\left\{\left[D_{\mu}, u^{\rho}\right] u_{\nu}\right\}+\frac{1}{2} \operatorname{Tr}\left\{\bar{B} \sigma^{\mu \nu} B\right\} \operatorname{Tr}\left\{\left[D_{\rho},\left[D_{\mu}, u^{\rho}\right]\right] u_{\nu}\right\}\right.$ \\
\hline & $\left.+\frac{1}{2} \operatorname{Tr}\left\{\bar{B} \sigma^{\mu \nu} B\right\} \operatorname{Tr}\left\{\left[D_{\mu}, u_{\rho}\right]\left[D^{\rho}, u_{\nu}\right]\right\}\right)$ \\
\hline 27 & $\frac{i}{2 m_{0}^{2}}\left(\operatorname{Tr}\left\{\bar{B} \gamma^{\mu}\left[\left[\left[D_{\mu}, u_{\nu}\right], u_{\rho}\right],\left[D^{\nu},\left[D^{\rho}, B\right]\right]\right]\right\}\right.$ \\
\hline & $\left.+\operatorname{Tr}\left\{\bar{B} \gamma^{\mu}\left[D_{\rho},\left[D_{\nu},\left[\left[\left[D_{\mu}, u^{\nu}\right], u^{\rho}\right], B\right]\right]\right]\right\}\right)$ \\
\hline 28 & $\frac{i}{2 m_{0}^{2}}\left(\operatorname{Tr}\left\{\bar{B} \gamma^{\mu}\left\{\left[\left[D_{\mu}, u_{\nu}\right], u_{\rho}\right],\left[D^{\nu},\left[D^{\rho}, B\right]\right]\right\}\right\}\right.$ \\
\hline & $\left.+\operatorname{Tr}\left\{\bar{B} \gamma^{\mu}\left[D_{\rho},\left[D_{\nu},\left\{\left[\left[D_{\mu}, u^{\nu}\right], u^{\rho}\right], B\right\}\right]\right]\right\}\right)$ \\
\hline 29 & $\frac{i}{m_{0}^{2}}\left(\operatorname{Tr}\left\{\bar{B} u_{\rho}\right\} \gamma^{\mu} \operatorname{Tr}\left\{\left[D_{\mu}, u_{\nu}\right]\left[D^{\nu},\left[D^{\rho}, B\right]\right]\right\}-\operatorname{Tr}\left\{\bar{B}\left[D_{\mu}, u_{\nu}\right]\right\} \gamma^{\mu} \operatorname{Tr}\left\{u_{\rho}\left[D^{\rho},\left[D^{\nu}, B\right]\right]\right\}\right.$ \\
\hline & $\begin{array}{l}-\operatorname{Tr}\left\{\bar{B}\left[D_{\nu},\left[D_{\mu}, u^{\nu}\right]\right]\right\} \gamma^{\mu} \operatorname{Tr}\left\{u_{\rho}\left[D^{\rho}, B\right]\right\}-\operatorname{Tr}\left\{\bar{B}\left[D_{\mu}, u_{\nu}\right]\right\} \gamma^{\mu} \operatorname{Tr}\left\{\left[D^{\nu}, u_{\rho}\right]\left[D^{\rho}, B\right]\right\} \\
-\operatorname{Tr}\left\{\bar{B}\left[D_{\rho},\left[D_{\mu}, u_{\nu}\right]\right]\right\} \gamma^{\mu} \operatorname{Tr}\left\{u^{\rho}\left[D^{\nu}, B\right]\right\}-\operatorname{Tr}\left\{\bar{B}\left[D_{\mu}, u_{\nu}\right]\right\} \gamma^{\mu} \operatorname{Tr}\left\{\left[D_{\rho}, u^{\rho}\right]\left[D^{\nu}, B\right]\right\} \\
-\operatorname{Tr}\left\{\bar{B}\left[D_{\rho},\left[D_{\nu},\left[D_{\mu}, u^{\nu}\right]\right]\right]\right\} \gamma^{\mu} \operatorname{Tr}\left\{u^{\rho} B\right\}-\operatorname{Tr}\left\{\bar{B}\left[D_{\rho},\left[D_{\mu}, u_{\nu}\right]\right]\right\} \gamma^{\mu} \operatorname{Tr}\left\{\left[D^{\nu}, u^{\rho}\right] B\right\}\end{array}$ \\
\hline & $-\operatorname{Tr}\left\{\bar{B}\left[D_{\nu},\left[D_{\mu}, u^{\nu}\right]\right]\right\} \gamma^{\mu} \operatorname{Tr}\left\{\left[D_{\rho}, u^{\rho}\right] B\right\}-\operatorname{Tr}\left\{\bar{B}\left[D_{\mu}, u_{\nu}\right]\right\} \gamma^{\mu} \operatorname{Tr}\left\{\left[D_{\rho},\left[D^{\nu}, u^{\rho}\right]\right] B\right\}$ \\
\hline 30 & $\frac{1}{2 m_{0}}\left(\operatorname{Tr}\left\{\bar{B} \sigma^{\mu \nu} \gamma^{5}\left[u_{\mu},\left\{u_{\nu},\left[u_{\rho},\left[D^{\rho}, B\right]\right]\right\}\right]\right\}+\operatorname{Tr}\left\{\bar{B} \sigma^{\mu \nu} \gamma^{5}\left[D_{\rho},\left[u^{\rho},\left\{u_{\nu},\left[u_{\mu}, B\right]\right\}\right]\right]\right\}\right)$ \\
\hline
\end{tabular}




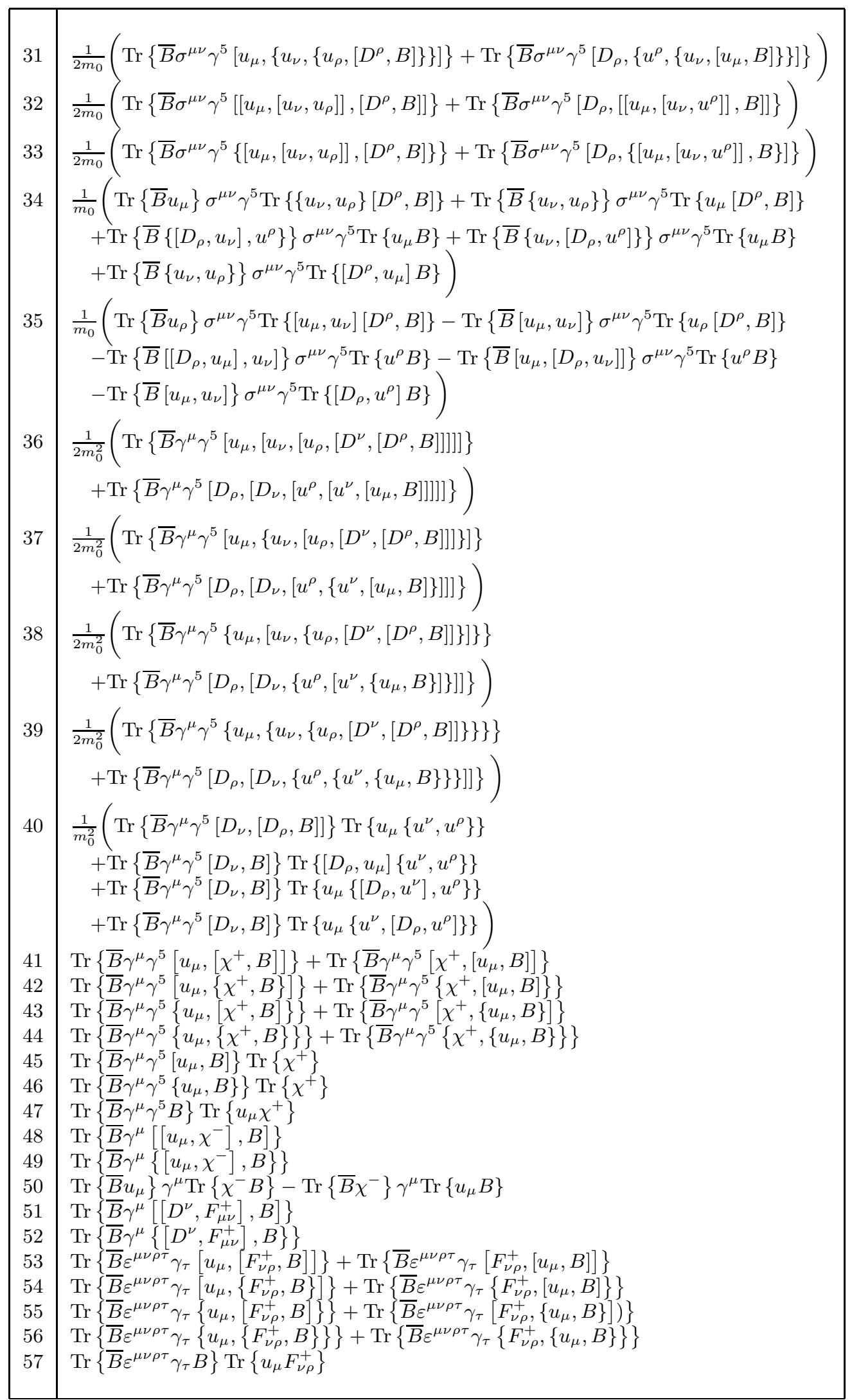




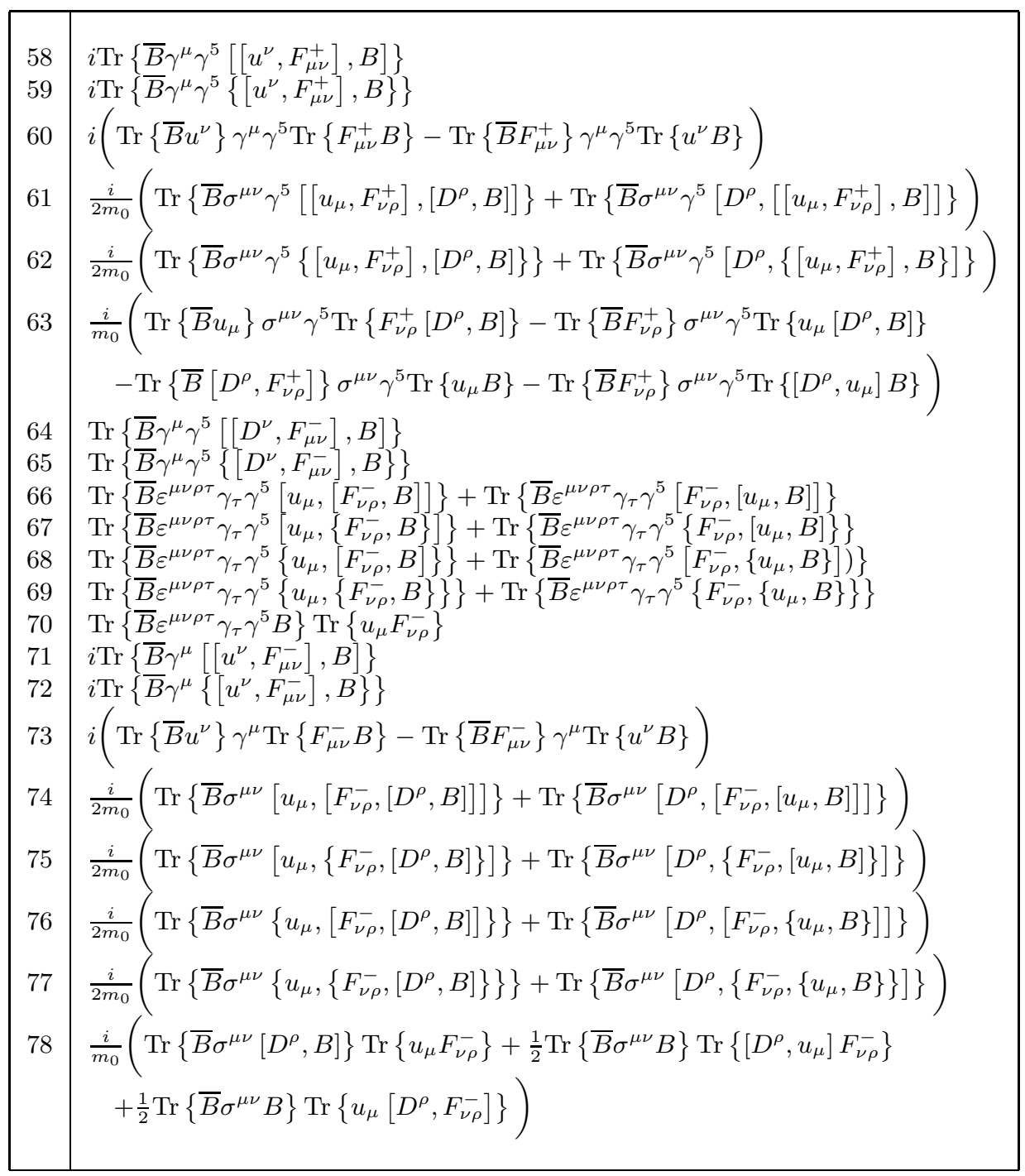

Table 1. Operators of $\mathcal{L}_{M B}^{(3)}$ For definitions, see Refs. [1,2]. 